\title{
A Novel Velocity-based Handoff Decision Policy for LTE Femtocell Networks
}

\author{
Aicha Salem \\ Innov'com, Supcom, Tunisie
}

\author{
K. Sethom \\ Innov'com, Supcom, Tunisie
}

\begin{abstract}
With the increased penetration of LTE and LTE-Advanced into the telecommunication market, many efforts have been made to increase the wireless coverage and extend it to previously inaccessible environments. Femtocells have been introduced in order to provide such coverage amelioration for regions where connectivity is reduced due to interference and signal attenuation issues. In this paper, we introduce a decision policy that takes into account the mobility aspect by considering the velocity of mobile stations and their cell-residence time in order to lessen excessive handoff and improve the overall network performance.
\end{abstract}

\section{Keywords:}

LTE, Handoff, Femtocell, velocity, energy, bandwidth

\section{INTRODUCTION}

The exponentially growing need for bandwidth coupled with the diverse QoS requirements have created a variety of technologies and standards. One of those standards is the Long Term Evolution of UMTS, which represents one of the latest steps of providing connectivity to mobile systems. Voice communication was, for a long time, the focus of mobile communication. With the birth of new multimedia services, the emphasis has shifted in favor of data transfer. The 3rd Generation Wideband Code Division Multiple Access (WCDMA) that have been chosen as the modulation of choice for $3 \mathrm{GPP}$ and its successor, known as Long Term Evolution are the culmination of effort to reach the goal stated in the previous paragraph. As the first specialized packet switched data service oriented, LTE had voice become just another data service thus bringing full realtime multimedia experience to reality. LTE is one of the leading technologies, as it targets the overall network throughput optimization while giving a special attention to QoS requirement. LTEAdvanced, a reiteration of LTE, improves user throughput and the global network capacity while allowing full mobility. The spectral resources are divided into several blocks named Resource Blocks (RB) or tiles. Those resource blocks are shared between the mobile stations and allocated using a scheduler, which role is to organize the access to the aforementioned blocks using several rules or heuristics. This strategy is centralized and, thus, may be globally optimal. Additionally, the diversity in the channel condition for each mobile user (multipath, fading, etc...) might have an adverse impact on the global performance of the network. A possible solution to this problem is the decentralization of scheduling, organizing the network into hierarchy, or both. LTE-advance provides such a solution: subdividing access points into two hierarchically distinct groups, which are macrocells and femtocells. Macrocell play the role of a classical access-point, while femtocell have either the role of an active relay, or behave as a wireless hub. Femtocells and the conventional macrocells are seen as isolated networks, with the only interaction between them is hand-over negotiations. Femtocells could also be seen as a network coverage extension tools, as their small size and low power consumption they can reach areas that were, in the past, inaccessible to base station such as inside metal reinforced concrete and metal sheet buildings. Femtocell access points are referred to as home NodeB (HNB) in Universal Mobile Telecommunications System (UMTS), according to the Third Generation Partnership Project (3GPP), and as Home eNodeB (HeNB) in LTE[1][2]. In the context of macrocell/femtocell networks, the handover decision could be decentralized or centralized, according to the scheduling and resource sharing policy. Femtocell Access Points (FAP) could be in the thousands for a handful of macrocell, which makes the handover decision even harder and more resource hungry[3]. In this paper we present a novel handover decision policy that should alleviate some of the classical bottlenecks and that is decentralized and takes into account the nature of the transmission channel (the multipath aspect mainly). This paper is divided into three sections. In the first section we will give a glimpse of the curret development in LTE and femtocell architectures. The second section will be dedicated to the formulation of the problem on hand and will be concluded by a mathematical model of our study case. The simulation and theoretical results will be the subject of the next section. This paper will be concluded by a discussion of the obtained results.

\section{LTE TECHNOLOGY}

We consider a mobile wireless cellular network in which mobile terminals and base stations implement an OFDMA air interface based on 3GPP/LTE downlink specification [4] [5]. Orthogonal Frequency Division Multiplex (OFDM) is the core of LTE downlink transmission [6] [7].

The OFDM symbols are partitioned into physical Resource Blocks (RBs), each consisting of 12 contiguous sub-carriers for 7 consecutive OFDM symbols. Therefore, $50 \mathrm{RBs}$ each having $10 \mathrm{MHz}$ are allocated for data transmission [8]. 


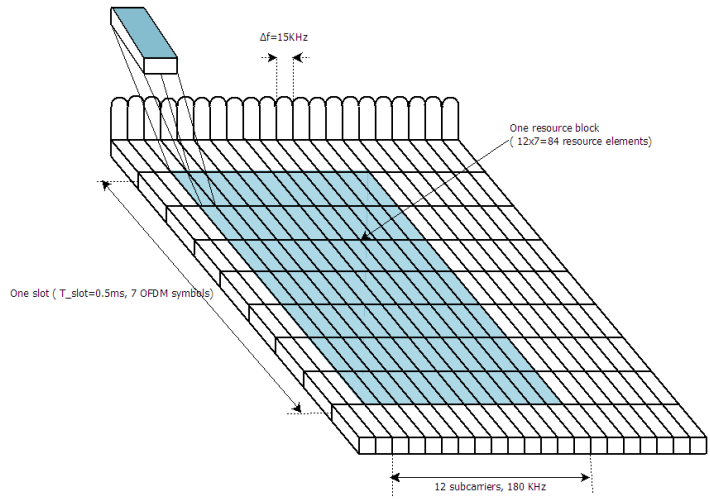

Fig. 1. LTE downlink physical resource grid

LTE downlink physical resource can be represented as a timefrequency resource grid as depicted in the Figure 1 [9]. The duration of a Resource Block (RB) is $0.5 \mathrm{msec}$ (one slot) and its bandwidth is $180 \mathrm{kHz}$ (12 subcarriers). It is a straightforward to see that each RB has $12 \times 7=84$ resource elements in the case of normal cyclic prefix and $12 \times 6=72$ resource elements in the case of extended cyclic prefix. The basic LTE downlink physical resource can be seen as a time-frequency grid [9].

\section{SYSTEM MODEL}

We consider one macrocell, with a BS located at the center of each hexagonal coverage area. M macro-users are randomly located inside the macro coverage area. The macrocell is deployed in an urban area, which is characterized by a dense building environment in addition to the increased mobility of the users, and coexists with femtocells.

Each femtocell provides service to its $\mathrm{N}$ associated femtousers. We consider that the total bandwidth $B_{w}$ is divided into subchannels which individual width is $\Delta f$. Orthogonal Frequency Division Multiplexing (OFDM) symbols are grouped into RBs [10].

Both macrocells and femtocells share the same frequency band and have the same amount $\mathrm{R}$ of available RBs, which allows to increases the spectral efficiency per area through spatial frequency re-use or in different band "dedicated channel". Fading would have, then, a positive impact as the deteriorated signal would not interfere greatly with the nearby station using the same RB. We focus only on the downlink operation. In this work we consider the case of hybrid mode where users are categorized according to their assigned priorities:

-P1: The highest priority, reserved for the femtocell owner; P1UE.

$-\mathrm{P} 2$ : The medium priority, assigned to other provider clients; $\mathrm{P} 2-$ UE.

-P3: the lowest priority level, to any guest user belonging to another provider for example; P3-UE.

Since frequency spectrum costs are high and spectrum is limited, the spectrum efficiency can be increased by higher reuse of the existing frequencies through cell-size reduction, from Macroto Femtocells with a macro overlay network. The topics to be addressed in future research are all connected to interferencelimited transmission with full frequency reuse.
These include CQI, QoS and interference-aware scheduling and RRM with user-grouping, coordinated RRM, exploiting the spatial degrees of freedoms from multi-antenna systems and FDMA through beamforming techniques, active interference management and CoMP transmission, as well as carrier aggregation for multi-band transmission. These topics have to be evaluated in the context of multi-user transmission in system-level environments.

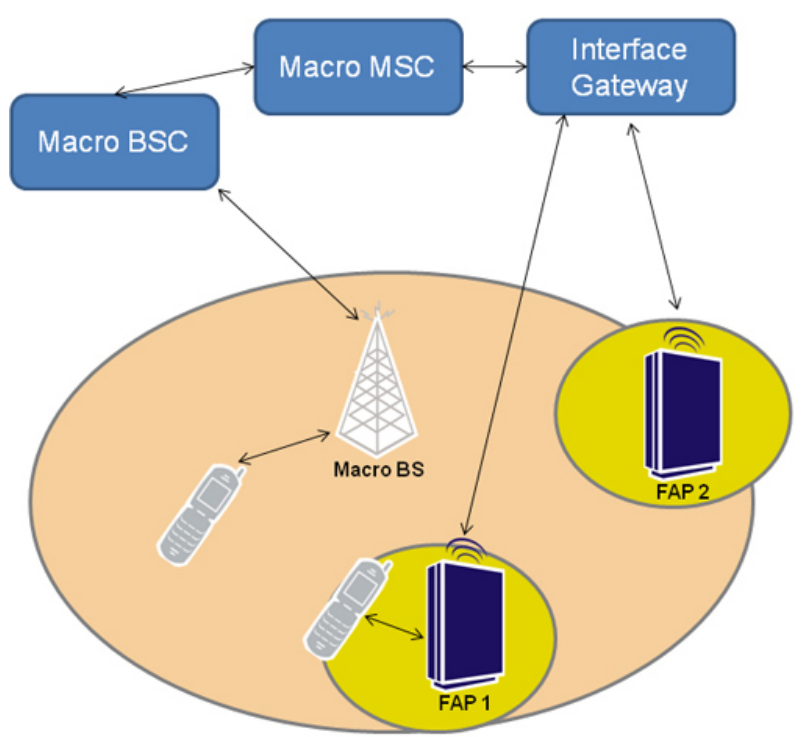

Fig. 2. Handover scenario in femtocell networks

To alleviate interference, one typical solution is to divide the entire available spectrum into several frequency bands and the femto-network use different frequency band other than those of the Macro-network (Figure 2). This deployment is referred to the "dedicated channel" deployment. However, the performance of this solution is limited by the assigned bandwidth, which makes it infeasible to be applied to the dense femto-networks deployment where each femto-network can only utilize a very limited bandwidth. As a result, a practical solution turns to be the co-channel deployment where femto networks and the Macronetwork share all available spectrum.

To mitigate interference in the co-channel deployment, dynamically power adapting schemes in femto-networks had been considered effective to alleviate interference. Another way to do is through dynamic resources allocation method in each femtocell: Given the total available bandwidth Bfem at the eNodeB, we associate to each class of priority users (P1, P2 and P3) its corresponding chunk of bandwidth derived from Bfem. i.e. B1 for P1 class, B2 for P2 class and B3 for P3. In a previous work [11], a game theory negotiation using Shapley value has been used to carry out a fair bandwidth distribution among priority classes.

A cooperative game is a game where groups of players ("coalitions") may enforce cooperative behavior, hence the game is a competition between coalitions of players, rather than between individual players. This discipline concerns the behavior of decision makers (players) whose decisions affect each other. A cooperative game consists of a player list and characteristic function. Given a set of players N, the players should form a coalition to transfer benefits among them [12]. 
In a bandwidth allocation game, the priority classes represent the players who benefits from capacity $\mathrm{C}$. All the classes form a coalition to get the benefit $C$. Under-loaded classes cooperates with overload classes, giving way unused capacity.

\section{CAPACITY METRIC}

The conventional way is through Received Signal Strength (RSS) .The RSSs are measured over time and the base station with strongest signal is chosen to handoff. Due to signal fluctuations, several handoffs may be requested while first BSs RSS is still sufficient to serve the mobile. These unnecessary handoffs are known as the ping-pong effect. As the number of handoffs increase, forced termination probability and network load also increases. Therefore, handoff techniques should avoid unnecessary handoffs. The second way is capacity-based handoff users connect to the cell that offers better capacity in terms of bandwidth [13]. In this paper, a handoff strategy that gives priority to how well the energy is being spent is introduced as the perenergy capacity based handoff.

\subsection{Capacity}

One of the main characteristics of the link between the base station and the users to be considered is the capacity. The capacity calculation is presented in different ways depending on: the bandwidth allocation strategy used, the users cell nature (macro or femtocell); and the users priorities. These definitions are mathematically expressed in (1)- (2) based on Shannon formula in mono-channel scenario. In co-channel operation strategy, the total bandwidth is shared $\left(B=B_{m a c}=B_{f e m}\right)$ and more interference is present. The capacities for users connected to femtocell $(\mathrm{Cf})$ and macrocell $(\mathrm{Cm})$ respectively are calculated by:

$$
\begin{gathered}
C_{f}=\frac{B_{f e m}}{N} \log \left(1+\frac{N P_{f}}{I_{f}+I_{m a c}+B_{f e m} \frac{T_{0}}{N}}\right) \\
C_{m}=\frac{B_{m a c}}{M} \log \left(1+\frac{N P_{f}}{I_{f e m}+B_{m a c j} \frac{T_{0}}{N}}\right)
\end{gathered}
$$

Where Imac and If are the interference coming from the macro station and surrounding femtocells (respectively) that affects the users connected to the femtocell, and Ifem is the interference that affects users connected to the macrocell coming from surrounding femtocells. $\mathrm{M}$ is the number of users connected to the macrocell.

\subsection{Velocity}

Given a mobile, traveling with an average speed $\mathrm{v}$ in a heterogeneous macro-femtocell environment for a distance $\mathrm{d}$, a valid macro/femto or femto-femto handover occurs if $\mathrm{v}$ is bounded by (5).Otherwise the mobile must remain attached to the macrocell:

$$
v<\frac{d\left(C_{f}-C_{m}\right)}{C_{f} L}
$$

where, $L$ is the handover latency.

Conventional handoff is not applicable when a mobile user is moving with a very high velocity [14]. As a user moves with a very high velocity it undergoes huge number of macro/femto handoff within a very short period of time. The overhead of the macro BS thus increases unnecessarily. Hence, in this paper we have considered a velocity threshold $V^{t h}$. Thus the unnecessary handoff is eliminated and improved QoS is guaranteed.

\section{PER-PRIORITY CAPACITY}

Lets now introduce user classes into the capacity evaluation. User with higher priority should be given better preference by the femtocell. Thats why; we change the femto capacity according to the following equation by adding priority class differentiation. When the roaming user priority is $P_{k}$ then:

$$
C_{f}=\sum_{j=1 \ldots k} \frac{P_{j}}{\sum_{i=1 \ldots P_{j}}} \times \frac{B_{j}}{N_{j}} \log \left(1+\frac{N_{j} P_{f}}{I_{f}+I_{m a c}+B_{j} \frac{T_{0}}{N}}\right)
$$

Where $N_{j}$ is the number of connected Pj-UE to the femtocell, and $\mathrm{Bj}$ the chunk of bandwidth dedicated to Pj class. Each term in the addition is moderated by the priority of user-class so that to give more importance to $\mathrm{P} 1$ users than $\mathrm{P} 2$ and respectively $\mathrm{P} 3$. In this way, we considered a context-aware handover selection method because the metric $\mathrm{C}$ is multi-criteria parameter from terminal and network sides. (6) reflects macro-to-femto and femtoto-femto interferences, bandwidth capacity, user priority according to the femtocell (the owner of the femto will be preferred to other client) and energy consumption.

However, for the macro-cell the capacity remains unchanged:

$$
C_{m}=\frac{B_{m a c}}{M} \log \left(1+\frac{P_{m}}{I_{f e m}+B m a c j \frac{T_{0}}{M}}\right)
$$

The proposed solution could be used as well for femto-femto handoff or Macro-femto handoff. The user has to compare simply the capacity (4) and (6) in case of a vertical handoff.

\subsection{Decision}

Let a mobile be connected to a source network, entering the wireless range of a Candidate base station (C). In this heterogeneous scenario, we model the decision metric between the two time instants tin and tout, as a positive range function $\beta$, defined as:

$$
B=\alpha \times\left(C_{c}-\lambda\right) \times(\Delta T-L)+(1-\alpha) \times C_{s} \Delta T
$$

Where $\alpha$ is an indicator function, such that $\alpha=1$ when a handover is executed, and zero otherwise. $\Delta T$ is the cell residential time. Since during the traveling time of a mobile it is desirable to maximize capacity, our technique initiates an handover only when it is valid, that is when $\beta_{\alpha=1}>\beta_{\alpha=0}$ and so when the inequality:

$$
C_{c}>\frac{C_{\text {source }}}{1-\frac{L}{\Delta T}}+\lambda
$$

In (7) $\lambda$ is a hysteresis factor introduced to avoid handover occurrence when the two competing networks have negligible capacity difference. From (7), we note how switching decisions may not be necessary even though the bandwidth $\mathrm{BCN}$ is higher than BSN. Switching becomes necessary only if the time that the mobile will spend in the cell with higher bandwidth is long enough to compensate for the data loss due to the switching overhead, namely, only if $L<\Delta T$ holds. This observation leads to the conclusion that the throughput is influenced not only by the bandwidth of the considered technologies, but by a larger set of 


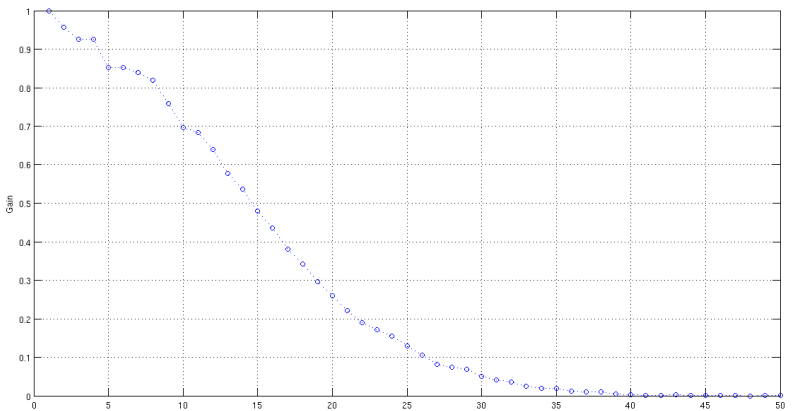

Fig. 3. Gain versus Velocity

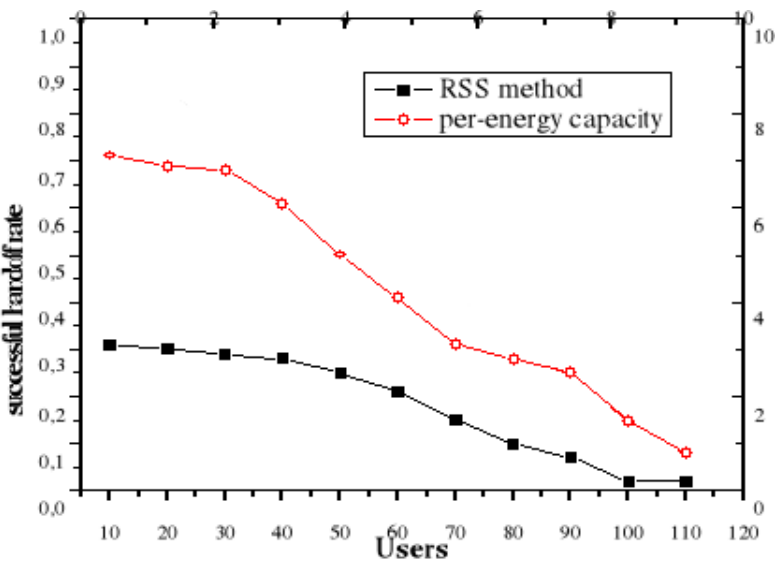

Fig. 4. Successful handover rate

parameters: the crossing time, the vehicle speed, and the overhead of the control-plane protocols adopted (handover latency) and interference.

Figure 3 shows the gain in function of velocity, using a Montecarlo simulation, and we can clearly see that the gain diminishes when the velocity increases. A low gain, in our case, means that no handoff should take place, as the mobile station is almost stationary. In higher velocities, handover becomes a necessity, as the mobile station would be leaving the coverage area soon. But, if the velocity is too high, then theres no need to begin the handover process, as it may last more than the time necessary to leave the coverage area.

\section{SIMULATIONS}

In this section, we assess the effectiveness of the proposed perpriority capacity. We consider a scenario in which 30 cellular users are deployed in the macrocell area. At the beginning of each run, users are randomly deployed in the macrocell region. Figure 4 shows the impact of the per-energy capacity with user priority calculation on the handover behavior. A successful handover is considered as so if the user connect to a new femtocell while he is satisfied by the offered QoS (bandwidth, interference, energy). The new proposed approach is better than conventional one that is only based on RSS level.

In figure 5 note that for our decision criterion, the throughput is noticeably better in function of wideband SINR than without it. This proves that optimizing the handoff decision to with-

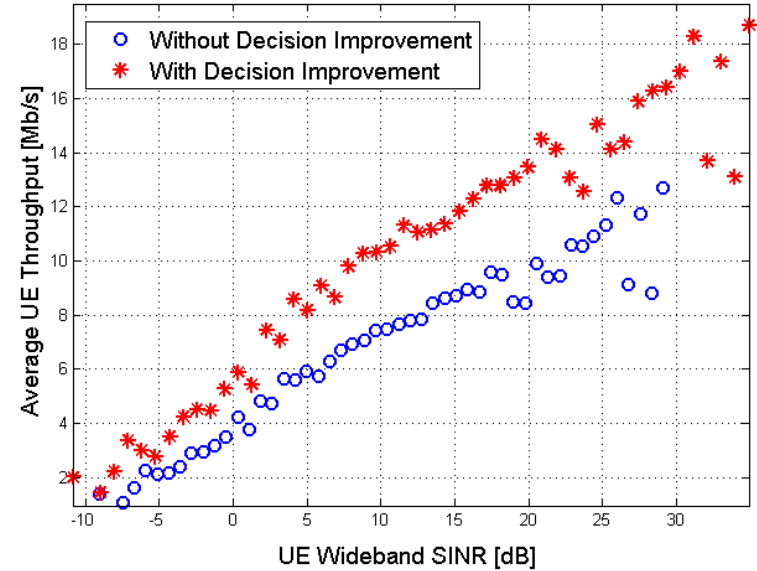

Fig. 5. Average UE throughput with and without decision improvement

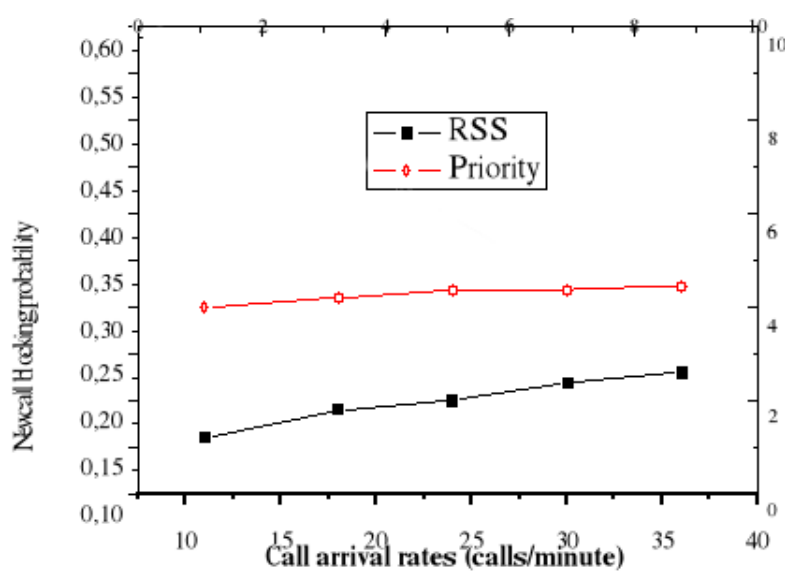

Fig. 6. New call blocking probability

stand variations within mobile station velocities is beneficial to the overall network performance.

In the next simulation (figure 6), we present the effect of the new capacity metric on new call admission. As you can see this of course regular best the system by blocking new calls and gives better priority to already connected users then classical schema based on RSS. The resource management of the networks is then best monitored.

\section{CONCLUSION}

In this paper, we have proposed a new velocity aware handoff strategy based on a novel metric that which reduces the unnecessary handoff in hierarchical macro/femto networks while improving the overall network performance throughput-wise. This implies a reduction in handoff related energy consumption while maintaining the QoS requirements.

\section{REFERENCES}

[1] H. Zhou, H. Luo, H. Zhang, C.H. Lo, H.C. Chao, A Network-based global mobility management architecture, In- 
ternational Journal of AdHoc and Ubiquitous Computing 2010 V. 4 (1) pp 82-95.

[2] L. Eastwood, S. Migaldi, Q. Xie, and V. Gupta,Mobility Using IEEE 802.21 in a Heterogeneous IEEE 802.16/802.11based, IMT-Advanced (4G) Network, IEEE Wireless Commun., April 2008.

[3] Guillaume de la Roche, Alvaro Valcarce, David Lpez-Prez, Jie Zhang, Access Control Mechanisms for Femtocells,IEEE COMMUNICATIONS MAGAZINE, JULY 2009

[4] K. Sundaresan, S. Rangarajan, "Efficient Resource Management in OFDMA Femto Cells," ACM International Symposium on Mobile AdHoc Networking and Computing (MobiHoc), May 2009.

[5] 3GPP- TS 23.401 v9.4.0, GPRS Enhancement for EUTRAN Access. 2010.

[6] YS Chen, CY Wu, A green handover protocol in two-tier OFDMA macrocell-femtocell networks, - Mathematical and Computer Modelling, 2012 Elsevier.

[7] YS Chen, CY Wu, A green handover protocol in two-tier OFDMA macrocell-femtocell networks, - Mathematical and Computer Modelling, 2012 Elsevier.

[8] LTE The UMTS Long Term Evolution: From Theory to Practice Stefania Sesia, Issam Toufik and Matthew Baker 2009 John Wiley and Sons.

[9] A. Ulvan, R. Bestak, M. Ulvan, The study of handover procedure in LTE- Based femtocell network, IEEE Wireless and Mobile Networking Conference, WMNC 2010, Budapest, Hungary, Oct. 2010, pp. 16.

[10] Assen Golaup, Mona Mustapha, and Leo Boonchin Patanapongpibul, Vodafone Group, Femtocell Access Control Strategy in UMTS and LTE, FEMTOCELL WIRELESS COMMUNICATIONS.

[11] Niyato D, Hossain E, Han Z. Dynamics of multiple-seller and multiple-buyer spectrum trading in cognitive radio networks: a game-theoretic modeling approach, IEEE Trans Mobile Comput 2009, 8(8):100922.

[12] K.Sethom et al., Resource management mechanism for femtocell enterprise networks IEEE Wireless advanced conference (Wiad) June 2012.

[13] Leon, Jaime Pena, and all. "Per-energy capacity and handoff strategies in macro-femtocells environment", 2012 IEEE Wireless Communications and Networking Conference Workshops (WCNCW), 2012.

[14] H. Zhang, X. Wen, B. Wang, W. Zheng, Y. Sun, A novel handover mechanism between femtocell and macrocell for LTE based networks, International Conference on Communication Software and Networks, ICCSN 2010, pp. 228231. 